\title{
Number theory and the unity of science
}

\section{AUTHORS:}

Jan C.A. Boeyens ${ }^{1}$

J. Francis Thackeray ${ }^{2}$

\section{AFFILIATIONS:}

${ }^{1}$ Centre for the Advancement of Scholarship, University of Pretoria, Pretoria, South Africa

${ }^{2}$ Evolutionary Studies Institute, University of the Witwatersrand, Johannesburg, South Africa

\section{CORRESPONDENCE TO: \\ Francis Thackeray}

\section{EMAIL:}

francis.thackeray@wits.ac.za

\section{POSTAL ADDRESS:}

Evolutionary Studies Institute, University of the Witwatersrand, PO WITS, Johannesburg 2050, South Africa

\section{KEYWORDS:}

Golden Ratio; constants; physics; chemistry; biology

\section{HOW TO CITE:}

Boeyens JCA, Thackeray JF. Number theory and the unity of science. S Afr J Sci. 2014;110(11/12), Art. \# a0084, 2 pages. http://dx.doi. org/10.1590/sajs.2014/a0084

\section{(C) 2014. The Author(s).} Published under a Creative Commons Attribution Licence.
Within recent millennia, sentient representatives of the species Homo sapiens have explored science with a sense of curiosity. Currently there are schoolchildren, university students and academic researchers, in Africa and elsewhere, asking questions about relativity, mass, space, particles, waves, space-time and the nature of constants in the fields of mathematics, physics, chemistry and biology. ${ }^{1}$ Recently, questions have been raised about whether an irrational mathematical constant - designated by the Greek symbol $\Phi$ with a value of about 1.618 - can be related to a biological species constant $(\mathrm{T})$, based on morphometric analyses of modern mammalian skulls, and explored in the context of probabilities of conspecificity of Plio-Pleistocene hominin fossils..$^{2-4}$ We suggest that there is a strong case that this so-called 'Golden Ratio' (1.61803...) can be related not only to aspects of mathematics but also to physics, chemistry, biology and the topology of space-time. ${ }^{1}$

A convincing case for assuming a cosmic character of the Golden Ratio can be made based on the ubiquity of logarithmic spirals. Spectacular examples include the Whirlpool Galaxy (M51), ammonites, the shape of Nautilus shells, Hurricane Katrina and the distribution of planets, moons, asteroids and rings in the solar system (Figure 1). The logarithmic spiral is firmly related to the Fibonacci series and the Golden Ratio of number theory. A familiar aspect of Fibonacci spirals is the way they feature in botanical phyllotaxis, the shape of kudu (Tragelaphus strepsiceros) horns and the curvature of elephant tusks. Less well known is the way in which the crystallographic structure of DNA, stress patterns in nanomaterials, the stability of atomic nuclides and the periodicity of atomic matter depend on the Golden Ratio. ${ }^{1}$ Apart from the Golden Ratio, a second common factor among this variety of structures is that they all represent spontaneous growth patterns. The argument that this amazing consilience ('self-similarity') arises from a response to a common environmental constraint, which can only be an intrinsic feature of curved space-time, is compelling.

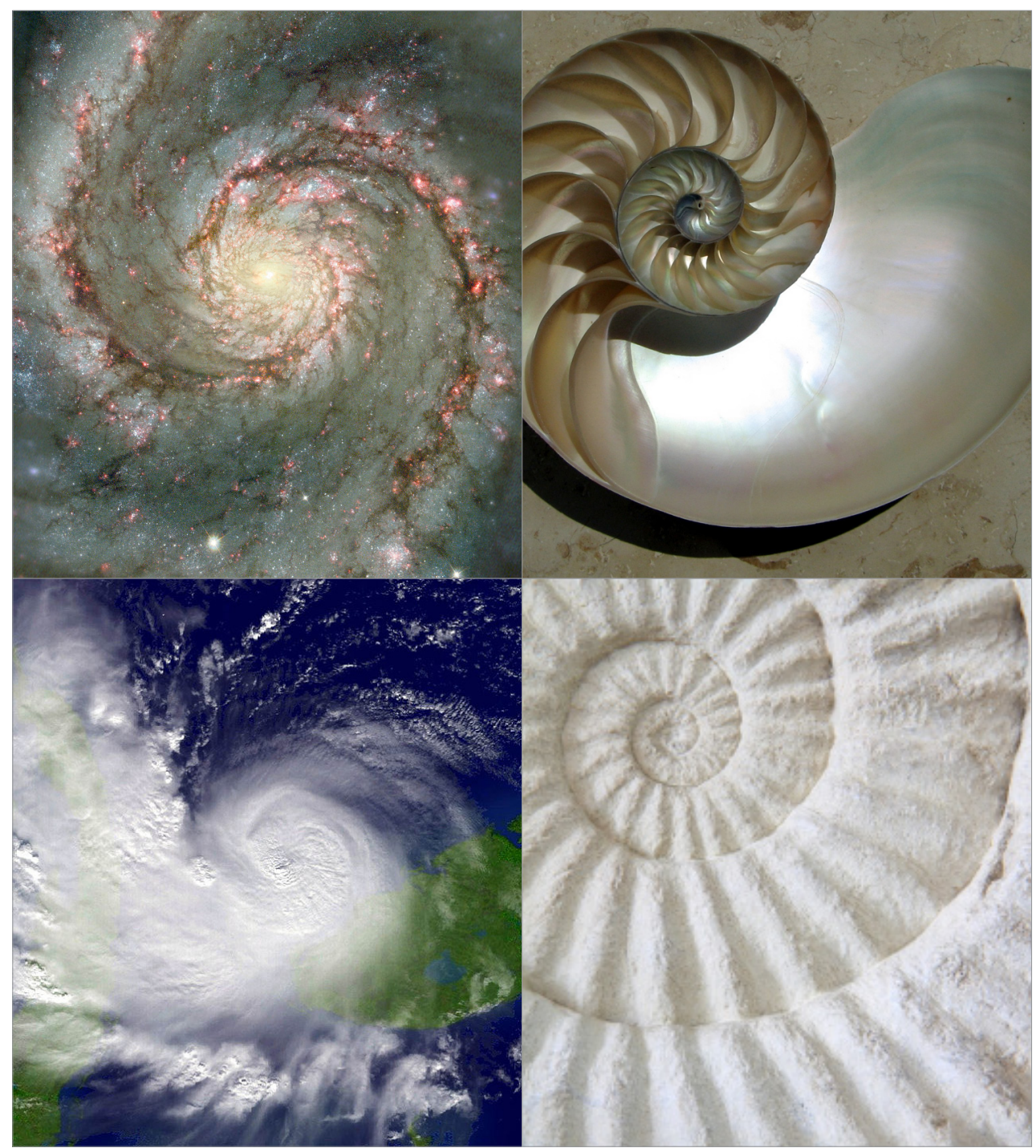

Sources: (left to right) NASA and The Hubble Heritage Team (Wikimedia Commons); Chris 73 (Wikimedia Commons); US National Oceanic and Atmospheric Administration (Wikimedia Commons); kongsky (FreeDigitalPhotos.net)

Figure 1: Examples of logarithmic spirals found in nature: (from left to right) the Whirlpool Galaxy, a Nautilus shell, Hurricane Katrina and an ammonite. 
In the context of biology, Thackeray ${ }^{3}$ has identified what he claims to be a species constant, based on the log-transformed standard error of the $m$-coefficient $\left(\log \mathrm{se}_{\mathrm{m}}\right)$, in regression analysis of cranial and other measurements from pairs of specimens of extant biological species (vertebrates and invertebrates), associated with regression equations of the form $y=m x+c$, where $m$ is the slope and $c$ is the intercept, using measurements of specimen $A$ (x-axis) and specimen $B$ of the same species (y-axis), or vice versa. Remarkably, an absolute mean $\log \mathrm{se}_{\mathrm{m}}$ value of ca 1.61 has been obtained for conspecific pairwise comparisons of extant vertebrates (mammals, birds, reptiles) and invertebrates (Coleoptera and Lepidoptera). ${ }^{3}$

An almost identical absolute mean log se value has been obtained from pairwise comparisons of conspecific Plio-Pleistocene hominin crania representing Australopithecus, Paranthropus or early Homo. ${ }^{5}$

An absolute mean $\log \mathrm{se}_{\mathrm{m}}$ value of 1.61 for pairwise comparison of dental measurements of hominin species has been calculated by Dykes ${ }^{6}$. In modern primates such as humans, chimpanzees, gorillas, orangutans and Colobus monkeys, the absolute mean log $\mathrm{se}_{\mathrm{m}}$ value for pairwise conspecific comparisons of crania is approximately $1.6 .^{7}$ The empirical evidence for a biological constant with central tendency of an absolute value of 1.61 is strong.

Transformation of the equations for relativistic quantum theory from curved space-time to Euclidean space coordinates was derived by Veblen and Hoffmann ${ }^{8}$ without realising the significance of the factor $\sqrt{5}=\Phi-1 / 2$ that correlates the electromagnetic potentials in the underlying curved space-time and tangent space. Examined more closely, the curvature of a Fibonacci spiral, $\pi / 2(2 \sqrt{2}) \simeq \sqrt{ } 5 / 2$, hence constitutes a convincing measure of space-time curvature.

Also demonstrated at an early date by Harkins ${ }^{9}$ was the fact that stable nuclides occur in a limited region defined by convergence to the proton:neutron ratio $(p / n)$ from unity to a value of 0.62 , later interpreted ${ }^{10}$ as $p / n=1 \rightarrow \tau$, where $\tau=1 / \Phi$. By the same reasoning, the remarkable observation that the structure of the periodic table of the elements is a function of environmental pressure ${ }^{11}$ can now be accounted for in detail, as a response to space-time curvature. The stability of atomic nuclides varies from $p / n=1$ at a black-hole singularity to $p / n=0.58$ in Euclidean space.
The time has come to recognise that relativity and quantum theories can be integrated, and linked numerically to the value of a mathematical constant - whether in the context of space-time ${ }^{1}$ or biology ${ }^{3}$.

\section{Acknowledgements}

This research is supported by the National Research Foundation (South Africa) and the Andrew W. Mellon Foundation.

\section{References}

1. Boeyens JCA. The chemistry of matter waves. Dordrecht: Springer; 2013. http://dx.doi.org/10.1007/978-94-007-7578-7

2. Thackeray JF. Probabilities of conspecificity. Nature. 1997;390:30-31. http:// dx.doi.org/10.1038/36240

3. Thackeray JF. Approximation of a biological species constant? S Afr J Sci. 2007;103:489.

4. Thackeray JF, Odes E. Morphometric analysis of early Pleistocene African hominin crania in the context of a statistical (probabilistic) definition of a species. Antiquity. 2013:87(335). Available from: http://antiquity.ac.uk/ projgall/thackeray335/

5. Thackeray JF. Palaeoanthropology: Probabilities of conspecificity. PaINews: Biannual Newsletter of the Palaeontological Society of Southern Africa. 2014;19(4):35-37.

6. Dykes SJ. A morphometric analysis of hominin teeth attributed to different species of Australopithecus, Paranthropus and Homo [MSc dissertation]. Johannesburg: University of the Witwatersrand; 2014.

7. Gordon AD, Wood BA. Evaluating the use of pairwise dissimilarity metrics in paleoanthropology. J Hum Evol. 2013;65:465-477. http://dx.doi. org/10.1016/j.jhevol.2013.08.002

8. Veblen 0, Hoffmann B. Projective relativity. Phys Rev. 1930;36:810-822. http://dx.doi.org/10.1103/PhysRev.36.810

9. Harkins WD. Periodic system of atomic nuclei and the principle of regularity and continuity of series. Phys Rev. 1931;38:1270-1288.

10. Boeyens JCA, Levendis DC. Number theory and the periodicity of matter. Dordrecht: Springer; 2008.

11. Boeyens JCA. Periodicity of the stable isotopes. J Radioanal Nucl Chem. 2003;257:33-43. http://dx.doi.org/10.1023/A:1024728806407 